ESJ Social Sciences

\title{
Enseignement et Formation : Deux Concepts Différents
}

\author{
Aguinou Lahoucine
}

Docteur en Sciences de l'Éducation, Maroc

\section{Doi:10.19044/esj.2022.v18n1p123}

Submitted: 18 October 2021

Accepted: 29 January 2022

Published: 31 January 2022
Copyright 2022 Author(s)

Under Creative Commons BY-NC-ND

4.0 OPEN ACCESS

Cite As:

Lahoucine A. (2022). Enseignement et Formation : Deux Concepts Différents. European Scientific Journal, ESJ, 18 (1), 123. https://doi.org/10.19044/esj.2022.v18n1p123

\section{Resume}

Cet article, qui s'inscrit dans le cadre des Sciences de l'éducation, tente de recenser les caractéristiques de l'enseignement et de la formation. Il met à la disposition des enseignants et des formateurs des connaissances nécessaires, afin d'éviter toute confusion entre les deux concepts. Pour réaliser les objectifs de cette étude, nous adoptons une méthode qualitative, suivant une démarche compréhensive, qui se base principalement sur la distinction pour nourrir la comprehension. Cette étude mobilise une recherche documentaire qui se base essentiellement sur les publications (ouvrages et articles scientifiques) qui traitent le sujet de cet article. Les données collectées sont traitées suivant une analyse inductive, guidée par les objectifs de la recherche. Cette méthode permet de définir et de comparer les sens, les pratiquants, les cibles, les modèles, les milieux de pratiques, les types, les méthodes et les outils d'enseignement et de formation. Les résultats de ce travail montrent que l'enseignement et la formation sont deux concepts différents, même si les praticiens les associent.

Mots-clés : Enseignement, Formation, Éducation, apprentissage 


\title{
Teaching and Training: Two Different Concepts
}

\author{
Aguinou Lahoucine \\ Doctor of Educational Science, Morocco
}

\begin{abstract}
This article, which falls within the framework of the Sciences of Education, attempts to identify the characteristics of teaching and training. It makes available to teachers and trainers the necessary knowledge to avoid any confusion between the two concepts. In order to achieve the objectives of this study, we adopt a qualitative method, following a comprehensive approach, which is mainly based on the distinction in order to nourish comprehension. This study mobilizes a documentary research which is essentially based on the publications (books and scientific articles) which deal with the subject of this article. The data collected are processed according to an inductive analysis, guided by the research objectives. This method makes it possible to define and compare the meanings, practitioners, targets, models, practice environments, types, methods and tools of teaching and training. The results of this work show that teaching and training are two different concepts, even if practitioners associate them.
\end{abstract}

Keywords: Teaching, Training, Education, learning

\section{Introduction}

Dans une ère de globalisation des échanges, les entreprises recourent aux formations, comme moyen de renforcer la performance, pour faire face à la concurrence, et aux exigences qui leur sont imposées. Aux pays développés, l'expression « la formation du personnel » est substituée par « la formation des compétences». Dans des économies concurrentielles, en perpétuelle évolution, les organisations exploitent leurs compétences pour assurer leurs performances. Elles investissent dans la formation des employés afin d'assurer la réalisation de leurs objectifs stratégiques. Au Maroc, il paraît que la situation est différente, notamment au secteur public, malgré les sommes colossales accordées à cette activité, qui s'inscrit dans le cadre de la gestion des ressources humaines. Il semble que les politiques gouvernementales n'accordent pas grande importance au développement des compétences des cadres. Les formations ne sont traitées que dans l'angle de l'exécution budgétaire. Leur qualité semble être le dernier souci des responsables. Au secteur d'éducation nationale, un grand nombre de formations est assuré, chaque année, par des personnes qui ne distinguent pas la formation de l'enseignement. 


\section{Les contours de la recherche \\ I.1. Problématique}

Aujourd'hui, nous vivons dans une société de savoir. L'information est abondante, ce qui dévoile le niveau des formateurs dans les domaines du savoir et du savoir-faire. Les bénéficiaires formés se sentent souvent insatisfaits. Ce qui justifie les faibles taux de réalisation des objectifs des formations, notamment dans le domaine de l'éducation. Les formations des éducateurs et éducatrices de l'enseignement préscolaire, organisées en 2020 et 2021, par le ministère de tutelle, ne présentent qu'un seul exemple de cette crise. Les conditions exigées pour réaliser les objectifs ne sont accomplies ni par les formateurs ni par les bénéficiaires. D'un côté, la plupart des formateurs sont obligés d'accomplir cette mission, n'ont bénéficié d'aucune formation dans ce domaine. De l'autre côté, les bénéficiaires recrutés par les associations, chargées de la gestion des classes de cet enseignement dans les écoles publiques, ne répondent pas aux conditions exigées par la loi, dans leur majorité. En conséquence de cette situation, certains formateurs ont été obligés de présenter des cours d'enseignement théorique, sous forme de PowerPoint, afin de passer les cinq longues journées de formation.

Ce qui précède nous incite à interroger les deux concepts : enseignement et formation. La pratique les associe, et les dictionnaires les différencient. Ils sont d'une importance égale. Ils renvoient à l'éducation et au développement des compétences. Ils se confondent au point où il est difficile de distinguer un cours présenté aux élèves, dans une classe, par un enseignant, d'un côté, d'une séance de formation présentée à un groupe de stagiaires, par un formateur, dans un centre de formation, de l'autre côté. Afin de mettre en lumière les deux concepts, cet article tente de répondre aux questions principales suivantes : que signifient l'enseignement et la formation? Quels sont les acteurs, les types, les objectifs, les programmes, les méthodes, et les outils d'enseignement et de formation?

\section{I.2. Hypothèses}

Afin de répondre aux questions posées, nous avançons les hypothèses suivantes :

- la définition de l'enseignement est différente de celle de la formation ;

- l'enseignement transmet des connaissances théoriques et la formation transmet des compétences pratiques ;

- les acteurs, les méthodes, les outils et les types de l'enseignement sont différents de ceux de la formation.

\section{I.3. Objectifs de la recherche}

Cet article, qui s'inscrit dans le cadre des Sciences de l'Éducation, vise à distinguer les concepts : enseignement et formation. Son but ultime est de 
mettre à la disposition des formateurs, les connaissances essentielles relatives à ces deux concepts, d'une manière simple, et détaillée, afin d'éviter d'éventuelles confusions conceptuelles. Il recense les caractéristiques de chacun des deux processus. Il présente les définitions, les acteurs, l'environnement des pratiques, les objectifs, les méthodes, les outils et les types d'enseignement et de formation.

\section{I.4. Méthodes et outils de collecte de données}

Pour réaliser ce travail, nous adoptons une méthode qualitative, suivant une démarche compréhensive. Cette méthode permet, d'une part, d'éviter les tensions entre la théorie et la pratique. De l'autre part, elle se base sur la distinction pour nourrir la compréhension.

Afin de collecter les données nécessaires pour mener à terme cet article, nous mobilisons une recherche documentaire, qui se base essentiellement sur les publications (ouvrages, articles scientifiques, etc.), traitant l'objet de l'étude.

Les données qualitatives collectées sont traitées suivant une analyse inductive, guidée par les objectifs de la recherche. Cette stratégie permet, à la fois, de définir et de comparer les sens, les pratiquants, les cibles, les modèles, les milieux de pratiques, les types, les méthodes et les outils d'enseignement et de formation.

\section{I.5. Le plan}

Nous commençons notre analyse par l'exposition des données globales collectées relatives à l'enseignement, qui concernent ses définitions, ses modèles, ses styles, ses méthodes, ses techniques et ses outils.

Nous enchaînons, ensuite, par la mise en lumière des éléments assemblés relatifs à la formation, qui présentent sa définition, ses contenus, ses milieux de pratiques, ses types, ses méthodes et ses outils.

Enfin, la conclusion présente un tableau de synthèse de la comparaison entre enseignement et formation.

\section{L'enseignement}

Dans ce qui suit, nous présentons des éclairages quant aux définitions, pratiquants, cibles, modèles, styles, méthodes et techniques d'enseignement.

\section{II.1. Quelques définitions}

«L'enseignement est un phénomène complexe » (Conseil de l'Europe, 2010 , p. 95), et une activité sociale et éducative. Il différencie de l'éducation. Ses définitions, qui sont multiples, s'accordent sur ses caractères : «mutuel », «interactif», et «intentionnel». Elles se basent sur l'implication des enseignants et des élèves dans une relation «sociale», «éducative» et 
« interpersonnelle », de « communication», d' «apprentissage» et de «transmission». Dans la conception classique, enseigner c'est faire des hommes, transmettre un message, transmettre un savoir. Enseigner c'est aussi apprendre, et créer des adultes dynamiques (LEM, s.d).

Généralement, sur le plan scientifique et conventionnel, «l'enseignement est un système d'actions visant à induire l'apprentissage » (Briguette, 2012, p. 141). Cette définition présente l'enseignement comme un système, composé d'un ensemble d'actions coordonnées et organisées à la fois de l'enseignant et de l'apprenant. Dans ce sens, J. Dewey, définit l'enseignement comme une "action entreprise avec intention d'apporter l'apprentissage à l'autre» (Briguette, Y-D, 2 012). Sur le plan pratique, "l'enseignement est un art au sens où il n'est pas dominé par des prescriptions et des routines, mais est influencé par des qualités et des contingences qui ne sont pas anticipées et se manifestent au cours de l'action » (OCDE, 2010, p. 48). Selon le dictionnaire de l'éducation de Legendre Larousse 1988, l'enseignement est un «processus de communication en vue de susciter l'apprentissage ». Il se définit également comme un processus institutionnalisé d'éducation et d'apprentissage systématique d'une première personne, qui s'appelle « enseigné » ou « élève », par une deuxième personne, présentée par l'enseignant. Il vise le développement des aptitudes, des habitudes et des attitudes des apprenants (Photios et al., 2002). Il est généralement théorique et s'appuie sur la transmission des connaissances et des concepts. Pour certains chercheurs, tels Amidon et Hunter, "l'enseignement est un processus interactif impliquant principalement l'interaction verbale qui a lieu en classe entre l'enseignant et les élèves, et ce, pendant certaines activités bien définies »(Briguette, 2012, p. 141). Cette définition présente l'enseignement comme un "processus ». Il est aussi une activité interpersonnelle et interactive liant l'enseignant et les élèves, dans une salle. Ces dernières définitions présentent la conception moderne de ce terme. Elles le traitent comme un concept plus large, qui intègre «l'organisation », «la facilitation » et «la gestion des apprentissages » (LEM, s.d).

À ces définitions, s'ajoutent celles d'Anderson et Burns, qui précisent que l'enseignement est une activité interactive interpersonnelle, qui implique une communication verbale, ayant comme finalité l'aide d'un ou plusieurs élèves à apprendre (Briguette, 2012).

\section{II.2. Pratiquant et ciblés d'enseignement}

Dans le système scolaire, les professeurs et les instituteurs sont des fonctionnaires qui forment le corps enseignant. L'enseignant est une personne, un agent et un travailleur et un maître qui maitrise les techniques d'enseignement. Il est un spécialiste de l'analyse qui arrive à développer chez les élèves les habiletés spécifiques attendues. Il est censé transmettre les 
apprentissages fondamentaux que les élèves doivent posséder. Il est un observateur, un évaluateur, et un animateur, qui aide, encourage, et accompagne ses apprenants. Les enseignants sont formés pour enseigner dans des classes de cours. Enseigner nécessite l'expertise d'un professionnel qualifié. "L'enseignant est un sujet-supposé-savoir et qu'il a un travail de transmission du savoir ». (Idrissi, 1982, p. 42). Il présente les caractéristiques de l'exemplarité d'un adulte. Il se présente comme un planificateur des conditions d'apprentissage, un facilitateur et un médiateur, qui assure l'accompagnement de ses élèves aux niveaux : psychologique et intellectuel.

Quant à l'élève, c'est un disciple qui reçoit l'enseignement. Il est un agent actif. «Il pense, il parle et il agit » (Collard, 1893, p. 532). Sa présence est souvent obligatoire.

\section{II.3. Milieu de pratique d'enseignement}

L'enseignement se déroule, souvent, dans un espace spécifique. Il s'exerce dans des salles de classe. Il se situe habituellement, dans le contexte de l'univers scolaire et universitaire. C'est ainsi qu' « il se réalise au sein d'une institution, l'école, qui se caractérise par plusieurs traits : hiérarchie des cycles de formation, séparation de la production, corps spécialisé d'enseignants, sanction de l'acquisition des savoirs par des promotions et par des diplômes. » (Photios et al., 2002, p. 264).

\section{II.4. Contenus d'enseignement}

Ce sont les enseignants qui éduquent les enfants à devenir de bons citoyens. Ils enseignent les contenus scolaires afin de stimuler l'apprentissage. Grâce aux concepts théoriques et connaissances transmises, les élèves peuvent faire face aux défis de leur entourage, et arrivent à intégrer leur société. Dans le contexte scolaire, «le programme scolaire est un instrument de contrôle des processus d'apprentissage institutionnalisés dans le cadre des établissements scolaires et comprend les objectifs, le contenu et les méthodes d'apprentissage» (Marques, 1979, p. 1). Il se définit aussi comme «un ensemble des matières et des sujets sur lesquels doit porter un enseignement. Nous le considérons comme un texte réglementaire et officiel qui sert de référence nationale pour fonder dans chaque discipline» (Guimfac, 2010, p. 27). Ce programme académique officiel est aussi appelé « curriculum ». C'est un contenu et un produit social, historique, standardisé et organisé. Il englobe des connaissances, des valeurs, des attitudes, des habiletés et des capacités qu'un apprenant est censé acquérir. Il met en relation les élèves et la société (Belinga, 2005). 


\section{II.5. Objectifs d'enseignement}

L'enseignement n'a pas pour objectif de rendre les apprenants performants. Il vise en plus du développement des aptitudes, des habitudes et attitudes des apprenants, l'enrichissement d'esprit, l'évaluation des acquis, la présentation d'un apprentissage de qualité, et la planification du soutien. Sur le plan économique, il fournit les travailleurs qualifiés (Touré, 2020), et crée les conditions propices à la créativité et au développement.

\section{II.6. Modèles d'enseignement}

Les modèles constituent le niveau le plus général de l'enseignement. Ils représentent ses orientations philosophiques. Ils permettent le choix de ses stratégies, ses méthodes, ses techniques, ses activités et ses situations pédagogiques appropriées (MAS, 1993, p.18).

Les modèles d'enseignement, les plus connus, sont :

- le modèle transmissif : il s'appuie sur l'hypothèse que l'apprenant ne sait rien du contenu enseigné, ou ne le connaît que de façon incomplète. L'enseignant cherche à remplir sa "tête vide», en lui expliquant un savoir, ou un savoir-faire. Ce modèle permet de gagner $\mathrm{du}$ temps. L'enseignant peut enseigner un groupe de personnes simultanément (Vianin, P, 2019, p. 230).

- le modèle "maïeutique scolaire»: il a été développé par le philosophe Socrate. Il se base sur l'hypothèse que toute personne a en elle-même la connaissance, car son âme a traversé le monde de la connaissance. L'enseignant aide l'élève à «accoucher» de la connaissance par un questionnement. Ce modèle présente des inconvénients au niveau de la gestion de la classe (Jacquet et Kambouchner, D. 2005, p.95).

- le modèle béhavioriste : il s'appuie sur les théories de psychologie, développées au début du XXe siècle. Il s'intéresse aux comportements observables, et correspond au conditionnement. Ce modèle a donné naissance à la " pédagogie par objectifs ». Il est centré sur l'apprenant (Le Thành, K, 1991, p.231).

- le modèle socioconstructiviste : ce modèle s'est développé contre le courant béhavioriste, amenant l'hypothèse que les comportements mentaux jouent un rôle fondamental dans l'étude du comportement humain et en particulier dans l'apprentissage. Il se concentre sur les compétences et les acquisitions des apprenants (Rege et Romainville, 2006, p. 217).

- le modèle de l'« apprentissage abstraction » : il est fondé par BraitMari Barth, s'inspire des recherches de Piaget et Bruner. Il est adapté aux concepts «classificateurs » seulement. Il met l'élève dans des situations complexes, ce qui lui permet de développer une démarche 
de recherche. Il rend l'acquisition des concepts possibles à travers l'appropriation de leurs caractéristiques. Quant à cette appropriation, elle s'effectue en trois étapes suivantes: la recherche des caractéristiques du concept enseigné, la représentation mentale et l'abstraction (Barth, 2013).

\section{II.7. Styles d'enseignement}

Selon le dictionnaire «ROBERT», le "style», est la "manière personnelle d'agir, de se comporter...». Le style d'enseignement approprié est identifié à partir du modèle adopté. Il montre la manière personnelle de l'enseignant de gérer sa relation avec ses élèves. En s'inspirant des travaux de Blake et Mouton (1964) en matière de management, Therer et Illemart distinguent quatre styles d'enseignement, qui sont :

- le style transmissif : il se centre uniquement sur la matière enseignée.

- le style incitatif : il se centre à la fois sur la matière et sur les apprenants.

- le style associatif : il se centre uniquement sur les élèves.

- le style permissif : il se centre beaucoup plus sur la matière que les apprenants.

Ces définitions combinent entre deux attitudes de l'enseignant. D'un côté, son attitude vis-à-vis de la matière, et de l'autre côté, son attitude vis-àvis des apprenants.

\section{II.8. Méthodes d'enseignement}

Elles se catégorisent en cinq groupes, qui sont :

- l'enseignement direct : c'est une méthode d'enseignement dirigée par l'enseignant, qui présente des informations, sous forme de cours magistraux, explicites et guidés aux élèves, dans une salle de classe.

- l'enseignement indirect : c'est un processus d'apprentissage centré sur les élèves. Ces derniers participent activement à ce processus en réalisant des recherches qui leur permettent de penser de manière critique, de prendre des décisions et de résoudre des problèmes. Ce type d'enseignement développe les compétences des élèves liées aux concepts enseignés.

- l'enseignement interactif : cette méthode est développée dans les années 1980, à l'Université de Waikato, dans le cadre du projet Learning in Science. Elle encourage la participation active des apprenants dans le processus d'apprentissage par le biais d'une interaction régulière (enseignant/élève, élève/élève), et en utilisant les moyens audiovisuels et des démonstrations pratiques. Elle offre des 
conditions de la compréhension et de l'utilisation de la mémoire à long terme.

- l'apprentissage expérientiel ou l'étude indépendante : cette méthode se base sur la théorie de l'apprentissage expérimental de Kob (1984). Elle traite les éléments clés de l'apprentissage contribuant à une pratique significative. Les enseignants l'utilisent pour soutenir la pratique de l'enseignement et les expériences des élèves. Il comprend quatre phases (l'expérimentation, l'observation réfléchie, la conceptualisation, et l'émission d'hypothèse).

\section{II.9. Techniques d'enseignement}

Les techniques représentent le niveau le plus spécifique des comportements d'un enseignant. Parmi ces techniques, nous citons: le questionnement, la discussion, les directives, les explications et les démonstrations. Il s'ajoute à ces techniques : la planification, la structuration, la focalisation, et la gestion.

\section{La formation}

Dans ce qui suit, nous mettons des lumières sur la définition, les pratiquants, les bénéficiaires, le contenu, le milieu de pratique, les types et les méthodes de la formation.

\section{III.1. Quelques définitions}

Le mot «formation» est employé en langue française depuis le XIe siècle, pour désigner le processus de transformation naturelle ou culturelle, permettant aux choses de prendre leurs formes finales, ainsi que les résultats de ces changements (Fabre, 1992). Elle diffère des autres formes d'apprentissage. Au domaine éducatif, "la formation englobe toutes les activités éducatives auxquelles pourrait s'adonner l'adulte, qu'il s'agisse d'études générales ou professionnelles, de recyclage ou de perfectionnement, de matières pertinentes à son travail comme à sa vie de citoyen" (Ndilbe, 2016, p. 20). Elle est « un processus continu et global qui se poursuit dans les activités diverses de la vie quotidienne » (Ader, 1961, p. 45). Elle est toujours préparée et accompagnée, tant au niveau de l'individu, qu'au niveau de l'organisation. La formation est aussi définie comme l'action d'apprendre en faisant, et l'action de transmettre des compétences pratiques et des comportements spécifiques à une personne ou un groupe de personnes. Elle concerne l'individu dans ses côtés: psychologique, mentale, affective et somatique. Elle permet le passage d'un état à un autre (Besançon et al, 1994). Elle se base sur l'apprentissage et le partage, qui implique l'acquisition de connaissances, le perfectionnement de compétences, de concepts et de règles. C'est ainsi que «la formation peut se définir comme l'ensemble des actions 
capables de mettre les individus et les groupes en état d'assurer avec compétence leurs tâches actuelles ou celles qui seront confiées dans leur futur pour la bonne marche de l'organisation» (Ndilbe, 2016, p.22).

Ces définitions considèrent la formation comme un ensemble d'actions, de moyens, de techniques et de supports planifiés qui visent l'amélioration des connaissances et des comportements, susceptibles de rendre les individus et les groupes capables d'assurer leurs fonctions avec compétence.

Au domaine du management, généralement, la formation est un ensemble de mesures adoptées en vue d'acquérir ou de perfectionner la qualification professionnelle des bénéficiaires (Aguinou, 2018). Elle est aussi un élément de la gestion des ressources humaines, qui est au service de l'amélioration du rendement, la mise en œuvre des stratégies de développement de la logique compétence de l'organisation. Elle anticipe l'évolution des métiers (Galambaud, 1993), en permettant le développement systématique de connaissances, aptitudes et compétences que demande l'exercice d'une tâche spécifique.

Dans le domaine économique, la formation est un moyen de résolution de problèmes (Hosdey et Rogester, 2009), de déblocage des situations (Palazzeschi, 1998)., d'ajustement, de perfectionnement et de promotion professionnelle par l'acquisition de nouvelles compétences, qui aide les chômeurs à retrouver des emplois. Elle permet aux bénéficiaires de satisfaire les exigences nécessaires pour exercer un métier. Elle aide également les employés à s'adapter au poste occupé, à évoluer vers d'autres fonctions, et à combler des retards de leur niveau, en général. Pour les entreprises, elle est un investissement (Camut, 1975), susceptible d'accroître la productivité et le rendement. Toute dépense visant l'amélioration des connaissances n'est pas une consommation. Cette thèse a été défendue par la théorie du capital humain, fondée par les économistes Schultz et Becker, au début des années 1960. Pour ces chercheurs, le niveau de formation des travailleurs d'un pays a un impact sur le niveau de ses richesses. La théorie des ressources internes a également encouragé les entreprises à investir dans le développement des stratégies de formation et de fédération des ressources humaines. Parmi les chercheurs qui ont appuyé cette théorie, nous citons : Cappelli, Crocker-Hefter, Lepak Wright et Snell.

\section{III.2. Pratiquants et bénéficiaires de formation}

La formation professionnelle, guidée par des formateurs émérites qualifiés et chevronnés, est susceptible de préparer les nouveaux employés à répondre aux exigences de l'emploi. Le formateur est un expert et un professionnel compétent qui a une compréhension approfondie des concepts théoriques et une expérience pratique. Il est un entraîneur qui facilite 
l'apprentissage et l'auto-apprentissage. Il possède les concepts théoriques nécessaires à la mise en ouvre des situations concrètes. Il transmet des connaissances et partages des compétences pratiques. Il connaît le terrain et possède l'expertise nécessaire pour offrir une formation aux stagiaires et aux employés.

La formation peut être offerte aux chômeurs, employés et aux stagiaires sélectionnés, accomplissant les conditions exigées, par l'organisateur. Elle vise, d'une manière égale, toutes les catégories de personnes, sans distinction d'âge, de niveau ou de responsabilités. Chaque formation a ses propres visées, qui sont souvent volontaires.

\section{III.3. Milieu de pratique de formation}

La formation est associée à la pratique au monde d'emploi. Elle se déroule, généralement, dans un environnement spécifique, au milieu du travail, ou dans les centres de formation, en dehors de ce milieu.

\section{III.4. Programmes de formation}

La formation est une application pratique des savoirs. Elle a pour objet ultime de transmettre un savoir, et un savoir-faire, par un formateur, à une personne ou à un groupe de personnes. Le programme de formation est bien planifié par l'organisateur pour atteindre des objectifs précis, en se basant sur des compétences spécifiques, exigées par le travail.

\section{III.5. Objectifs de formation}

La formation prépare ses bénéficiaires à faire face aux changements. Elle améliore leurs employabilités grâce à la transmission des compétences pratiques nécessaires à l'intégration socioprofessionnelle. Elle développe leurs compétences techniques pour mener à bien leurs missions. Elle permet aux employés de conserver leur emploi et assure leur perfectionnement et leur progression au sein de leurs organisations. Sur le plan psychologique, la formation accroît la motivation des employés, qui se sentent reconnus par leur employeur. Elle procure également un sentiment de fierté après l'obtention du certificat ou du diplôme de formation (Hosdey et Rogester, 2009). Sur le plan économique, la formation, comme moyen de croissance économique, permet aux organisations d'améliorer leur efficacité et leur rentabilité en matière de production.

\section{III.6. Types de formation}

Les types de formation les plus connus sont :

- la formation initiale : elle se présente en présentiel et sans alternance, et constitue, habituellement, une continuité d'un parcours scolaire. 
- la formation continue : elle s'oriente vers les personnes désireuses de changer d'emploi, ou de perfectionner leur expérience.

- la formation professionnelle : elle a pour objet d'acquérir de nouvelles compétences, susceptibles d'aider la personne formée à évoluer dans son parcours professionnel, ou à changer sa profession.

- la formation en alternance : son principe est d'alterner entre les périodes de cours et de formation théorique et périodes de travail. Elle permet aux stagiaires de se former gratuitement.

- la formation en présentiel : c'est une formation dont l'interaction du formateur et ses cibles se déroulent au sein du même lieu. Elle se caractérise par un accompagnement constant du formateur, qui répond directement aux besoins des apprenants.

- la formation à distance : elle est flexible et ne s'effectue pas en présentiel. Elle concerne les personnes désireuses de suivre les contenus de la formation, depuis chez elles. Les moyens utilisés par le formateur, dans son accompagnement, sont variés (téléphone, platesformes en ligne, instruments numériques, réseaux sociaux, courriels, supports papier par voie postale, etc.).

- la formation de type blende Learning : elle associe les deux types de modalités pédagogiques (présentiel et à distance).

- la formation en ligne : elle correspond à la formation via internet, via les plates-formes.

\section{III.7. Méthodes de formation}

Les méthodes de formation sont celles employées pour communiquer les contenus et permettre les apprentissages. Qu'elles soient formelles ou informelles, elles ont toutes pour but de faciliter la transmission des savoirs, de savoir-faire et de savoir-être. Le choix de la méthode appropriée est conditionné par le choix du style d'apprentissage adopté (convergent, divergent, assimilateur ou accommodateur). Les travaux de: Bazin, Fernandez, Laflamme, Meignant, Mucchielli, et Sonntag distinguent trois groupes de méthodes de formation, qui sont :

- les méthodes affirmatives : elles se basent sur les formateurs qui détiennent le savoir.

- les méthodes interrogatives : elles considèrent les formateurs comme des maîtres. Ces derniers recourent au questionnement, considéré moyen de développement des connaissances et compétences des bénéficiaires. Ces derniers se situent dans des situations actives de recherche de réponses.

- les méthodes actives : elles se basent sur l'implication active des personnes formées dans le processus d'apprentissage, aux niveaux : 
intellectuel, affectif et émotionnel. Elles n'exigent pas la possession du savoir par le formateur. Ce dernier ne joue que le rôle de guide et de facilitateur. Son intervention est très limitée.

\section{III.8. Outils de formation}

Ce sont des instruments techniques que les formateurs utilisent pour réussir leurs formations. Le choix de ces outils est conditionné par certains facteurs, tels que: les objectifs, le coût, l'interaction des intervenants, les activités planifiées de la formation. Généralement, les outils de formation se répartissent en quatre catégories suivantes :

- outils visuels : parmi ces outils, nous citions : le tableau, le rétroprojecteur et les notes de cours.

- outils auditifs : le magnétophone et le téléphone sont les outils de ce type les plus connus.

- outils audiovisuels : parmi ces moyens, nous citons : le diaporama, le film et le magnétoscope.

- outils interactifs : ce sont des outils qui permettent l'interaction, comme : l'ordinateur, le didacticiel et le simulateur.

\section{Conclusion}

Les résultats de notre recherche documentaire confirment toutes les hypothèses de la recherche. Ils montrent que la formation et l'enseignement sont deux concepts différents, même s'ils renvoient aux pratiques d'éducation, visant l'acquisition des connaissances et le développement des compétences des bénéficiaires, d'une manière académique.

Le tableau suivant résume les différences entre les deux concepts.

Tableau1 : Synthèse de la comparaison entre l'enseignement et la formation

\begin{tabular}{|c|c|c|}
\hline & Enseignement & Formation \\
\hline Définitions & $\begin{array}{l}\text { - un phénomène complexe } \\
\text { - une action sociale éducative } \\
\text { interactive et intentionnelle } \\
\text { - un système } \\
\text { - un art } \\
\text { - un processus de communication, de } \\
\text { transmission et d'apprentissage. }\end{array}$ & $\begin{array}{l}\text { - un élément de la GRH } \\
\text { - un ensemble d'actions } \\
\text { - un ensemble de mesures, de moyens, } \\
\text { de techniques et de supports planifiés } \\
\text { - un investissement } \\
\text { - une solution des problèmes de } \\
\text { l'organisation. }\end{array}$ \\
\hline Acteurs & $\begin{array}{l}\text { - les enseignants } \\
\text { - les élèves }\end{array}$ & $\begin{array}{l}\text { - les formateurs expérimentés } \\
\text { - les experts- les entraîneurs- les } \\
\text { stagiaires - les employés -les } \\
\text { chômeurs- etc. }\end{array}$ \\
\hline Environnements & $\begin{array}{l}\text { - un espace spécifique et contrôle } \\
\text { - une salle de classe }\end{array}$ & $\begin{array}{l}\text { - le milieu du travail } \\
\text { - les centres de formation } \\
\text { - l'environnement qui garantit une } \\
\text { certaine liberté }\end{array}$ \\
\hline
\end{tabular}




\begin{tabular}{|c|c|c|}
\hline Objectifs & $\begin{array}{l}\text { - l'apprentissage des concepts } \\
\text { théoriques } \\
\text { - le changement des représentations }\end{array}$ & $\begin{array}{l}\text { - la transmission des compétences } \\
\text { pratique } \\
\text { - le changement des pratiques } \\
\text { - l'amélioration de l'employabilité } \\
\text { - le perfectionnement et la progression } \\
\text { au travail } \\
\text { - l'amélioration de l'efficacité et de la } \\
\text { rentabilité } \\
\end{array}$ \\
\hline Méthodes & $\begin{array}{l}\text { - l'enseignement direct } \\
\text { - l'enseignement indirect } \\
\text { - l'enseignement interactif } \\
\text { - l'apprentissage expérientiel } \\
\text { - l'étude indépendante }\end{array}$ & $\begin{array}{l}\text { - les méthodes affirmatives } \\
\text { - les méthodes interrogatives } \\
\text { - les méthodes actives }\end{array}$ \\
\hline Outils & $\begin{array}{l}\text { - le questionnement } \\
\text { - la discussion } \\
\text { - les directives } \\
\text { - les explications } \\
\text { - les démonstrations } \\
\text { - la planification } \\
\text { - la structuration } \\
\text { - la focalisation } \\
\text { - la gestion. } \\
\end{array}$ & $\begin{array}{l}\text { - les outils visuels } \\
\text { - les outils auditifs } \\
\text { - les outils audiovisuels } \\
\text { - les outils interactifs }\end{array}$ \\
\hline Types & $\begin{array}{l}\text { - le style transmissif } \\
\text { - le style incitatif } \\
\text { - le style associatif } \\
\text { - le style permissif }\end{array}$ & $\begin{array}{l}\text { - la formation initiale } \\
\text { - la formation continue } \\
\text { - la formation professionnelle } \\
\text { - la formation en alternance } \\
\text { - la formation en présentiel } \\
\text { - la formation à distance } \\
\text { - la formation blende Learning } \\
\text { - la formation en ligne }\end{array}$ \\
\hline
\end{tabular}

\section{References:}

1. Ader, J. (1961). Processus du perfectionnement des connaissances et de la formation personnelle chez l'adulte : rapport de la réunion des 8-13 février 1960. UNESCO : Institut de l'UNESCO pour l'éducation

2. AGUINOU, L. (2018). La madrassa au Maroc: entre tradition et modernité. Cas de la madrassa de Sidi Ouaggag, CT d'Aglou, province de Tiznit (2012-2017). Thèse, Faculté des Sciences de l'Éducation, Université Mohamed V, Rabat, Maroc.

3. Barth, B-M. (2013). L'apprentissage de l'abstraction. Paris : Editions Retz

4. Belinga, B-S. (2005). Didactique et professionnalisation des enseignants. Yaoundé : Éditions CLE

5. Besançon, J. Maubant, Ph. Ouzilou, C. (1994). L'individualisation de la formation en questions. Délégation à la formation professionnelle. France : La Documentation Française - Collection Recherche en Formation Continue 
6. Briguette, Y-D, (2012). La taille des classes et la qualité des procédures dans l'enseignement primaire centrafricain : théorie et pratique, Cas pratique : comprendre et évaluer. Paris : Publibook

7. Camut, R. (1975). La formation générale des ouvriers vue par un ouvrier. Paris : Entreprise Moderne d'Édition

8. Collard, F. (1893). Le gymnase et le séminaire pédagogique de Giessen. Revue internationale de l'Enseignement, V25, pp. 525-544. Paris : Armand Colin et Cie Éditeur. URL : https://education.persee.fr/doc/revin_17756014_1893_num_25_1_2958

9. Conseil de l'Europe, (2010). Politiques et pratiques de l'enseignement de la diversité socioculturelle. Strasbourg: Edition du conseil de l'Europe

10. Fabre, M. (1992). Qu'est-ce que la formation? Recherche \& formation, (12), pp.119-134. URL : https://www.persee.fr/doc/refor_09881824_1992_num_12_1_1115

11. Galambaud, B. (1993). Le développement social : Les pratiques de mobilisation dans l'entreprise. Les Cahiers français. Paris : Éditions de la Documentation française, Numéro 262, juill.-sept. p.39-71

12. Guimfac, M. (2010). L'enseignement-apprentissage de la lecture au collège en Afrique noire - Cas de quelques collèges de Brazzaville. France : L'Harmattan.

13. Hosdey, A. et Rogester, J. (2009). La formation en entreprise : les clés de la réussite. Belgique : Edipro

14. Idrissi, A, (1982). Maitrise pédagogique et désir de pouvoir. Lamalif, $\mathrm{n}^{\circ}$ 136. URL : http://bnm.bnrm.ma:86/pdf.aspx?IDc=2735

15. Le Thành, K. (1991). L'éducation: cultures et sociétés. Paris, Publications de la Sorbonne.

16. LEM, (s. d). Styles d'enseignement, styles d'apprentissage et pédagogie différenciée en sciences. Liège: Laboratoire d'Enseignement Multimédia (LEM) de l'Université de Liège. URL : http://www.lmg.ulg.ac.be/competences/chantier/eleves/lem_art2.html (consulté le 13 avril 2021)

17. Marques, J. H. F. (1979). Orientation et programme scolaire. Paris : Unesco.

URL : http://books.google.com/books?id=hLElAQAAMAAJ.(consulter le 11 septembre 2021)

18. Ministère de l'Apprentissage de la Saskatchewan (MAS). (1993). Approches Pédagogiques: Infrastructure pour la pratique de l'enseignement. Saskatchewan: Gouvernement de la Saskatchewan. 
19. Ndilbe, N-H. (2016). Le management du personnel dans une société : le cas de Socatel. Mémoire-Licence, Université de Bangui - Licence, la République centrafricaine

20. Jacquet-Francillon, F. Kamboucher, D. (2005). La crise de la culture scolaire: Origines, interprétations, perspectives. Paris: Presses Universitaires de France.

21. OCDE, (2010). Comment apprend-on? La recherche et l'innovation dans l'enseignement. Paris : Éditions OCDE

22. Palazzeschi, Y. (1998). Introduction à une sociologie de la formation : Les évolutions contemporaines. France : L'Harmattan

23. Photios, G. et al., (2002). La Côte d'Ivoire à l'aube du XXIe siècle : défis démographiques et développement durable. Paris : Éditions KARTALA

24. Rege, N. Romainville, M. (2006). La pratique enseignante en mutation à l'université: perspectives en éducation et formation. Bruxelles : Deboek.

25. Touré, D. (2020). Enseignement supérieur, capital humain et croissance économique: Une approche par l'analyse causale. European Scientific Journal, ESJ, Vol.16, No.16. URL:http://dx.doi.org/10.19044/esj.2020.v16n16p255

26. Vianin, P.(2019). La supervision pédagogique: L'accompagnement des stagiaires. Collection Pratiques pédagogiques, 1ère édition. Bruxelles : DEBOEK. 